\title{
Association of Antituberculosis Treatment and Lower Risk of Hyperlipidemia in Taiwanese Patients: A Population-Based Case-Control Study
}

\author{
YING-RAY LEE ${ }^{*}$, NI TIEN ${ }^{2 *}$, CHENG-LI LIN ${ }^{3}$, HSIN-YI SHEN ${ }^{4}$, DA-TIAN BAU ${ }^{5,6}$ and YUN-PING LIM ${ }^{4,7,8}$ \\ ${ }^{1}$ Translational Medicine Research Center, Chia-Yi Christian Hospital, Chiayi, Taiwan, R.O.C.; \\ ${ }^{2}$ Department of Microbiology, Laboratory Medicine, China Medical University Hospital, Taichung, Taiwan, R.O.C.; \\ ${ }^{3}$ Management Office for Health Data, China Medical University Hospital, Taichung, Taiwan, R.O.C.; \\ ${ }^{4}$ Department of Pharmacy, College of Pharmacy, China Medical University, Taichung, Taiwan, R.O.C.; \\ ${ }^{5}$ Graduate Institute of Biomedical Sciences, China Medical University, Taichung, Taiwan, R.O.C.; \\ ${ }^{6}$ Department of Bioinformatics and Medical Engineering, Asia University, Taichung, Taiwan, R.O.C.; \\ ${ }^{7}$ Department of Internal Medicine, China Medical University Hospital, Taichung, Taiwan, R.O.C.; \\ ${ }^{8}$ Department of Medical Research, China Medical University Hospital, Taichung, Taiwan, R.O.C.
}

\begin{abstract}
The association between anti-tuberculosis (TB) treatments and the risk of developing hyperlipidemia remains unclear. Data were obtained from the Longitudinal Health Insurance Database 2000 (LHID2000). The case group included patients newly diagnosed with hyperlipidemia $(n=16,054)$ between 2006 and 2011 selected from the LHID2000. A four-fold number of hyperlipidemia-free cases $(n=64,216)$ were matched with case patients by age, sex, and index year to create the control group. Univariable and multivariable unconditional logistic regression analyses were conducted to estimate the odds ratios (ORs) and 95\% confidence intervals (CIs) for the association between hyperlipidemia and anti-TB medication use. Patients that used isoniazid (INH) were significantly associated with a decreased risk of hyperlipidemia $(O R=0.71,95 \% C I=0.57$ 0.88). After adjustment for age, sex, urbanization level, and income as well as ethambutol, pyrazinamide, streptomycin, and anti-human immunodeficiency virus drug medications, a dose-dependent risk of hyperlipidemia was observed in the
\end{abstract}

This article is freely accessible online.

*These Authors contributed equally to this study.

Correspondence to: Yun-Ping Lim and Da-Tian Bau, Department of Pharmacy, College of Pharmacy, China Medical University, No. 91, Hsueh-Shih Road, Taichung 40402, Taiwan, Republic of China. Tel: +886 422053366 ext. 5802, Fax: +886 422078083, e-mail: limyp@mail2000.com.tw, limyp@mail.cmu.edu.tw, artbau2@gmail.com

Key Words: Anti-tuberculosis, isoniazid (INH), rifampin (RIF), hyperlipidemia, population-based case-control study.
INH, rifampin (RIF), and INH and RIF groups with the ORs progressively decreasing as the cumulative dose increased. In the Taiwanese patients who used anti-TB medications, INH and RIF use was associated with a decreased risk of hyperlipidemia.

Tuberculosis (TB), caused by infection with Mycobacterium tuberculosis, remains a major public health disease worldwide (1). The World Health Organization (WHO) reported that, in 2015, an estimated 10.4 million people developed TB, half of whom $(58 \%)$ were found in Southeast Asia and the Western Pacific Region, and 1.4 million died from this disease worldwide (1). Because of improvements in medical treatment, the incidence of TB is declining annually, and an estimated number of people saved from TB decreased by $22 \%$ between 2000 and 2015 as a consequence (1). In addition, successful TB treatment has averted 49 million deaths between 2000 and 2015 globally (1). Although mortality rates for TB decreased by approximately $35 \%$ between 1990 and 2009, the number of cases and incidence rate fell $25.1 \%$ and $26.8 \%$, respectively, between 2005 and 2012. TB remains a serious infectious disease and is the 17 th leading cause of death in Taiwan $(2,3)$. Among people of older age or with a weakened immune system, the pathogenic microorganism M. tuberculosis can be reactivated, potentially leading to serious long-term pneumonia and lung scarring (4). If untreated, TB may cause chronic inflammation and has a mortality rate of $50 \%$ within 5 years (4).

According to current international guidelines, TB treatment consists of a two month intensive phase with 4 drugs (isoniazid [INH], rifampin [RIF], pyrazinamide, ethambutol) followed by a continuation phase of at least 4 months with two 
drugs (INH, RIF) (5). They are also used in combination with other medications to treat coinfections (6). However, a variety of adverse reactions to these drugs have been reported. The most well-known toxic effect is hepatotoxicity (7). Using these drugs in combination may increase the risk of hepatocellular carcinoma (HCC) in patients with liver cirrhosis (8).

Combinatorial therapy for INH and RIF induces reactive oxygen species (ROS) overproduction and hepatocyte damage (9). Chronic anti-TB treatments thus may intensify the imbalance of redox status and promote oxidative stress because of lipid deposition $(10,11)$. However, a comprehensive literature review has suggested that information pertaining to blood lipids and these anti-TB medications remains limited. We thus conducted a large, nationwide case-control study by using data from the Longitudinal Health Insurance Database (LHID) maintained by the National Health Research Institutes (NHRI) of Taiwan to assess the risk of hyperlipidemia associated with using INH and RIF among Taiwanese patients.

\section{Materials and Methods}

Data source. Datasets were obtained from the reimbursement database of the Taiwan National Health Insurance (NHI) program, a single-payer universal insurance system (8). This insurance system covers more than $99 \%$ of the 23.74 million residents of Taiwan (http://www.nhi.gov.tw/english/index.aspx). Claims data from the LHID, which was established by the NHRI and consists of the claims data of $1,000,000$ patients randomly sampled from the population of all NHI beneficiaries, were used. The distributions of sex, age, and health care costs are not significantly different between the cohorts in the LHID and all insurance enrollees, as reported by the NHRI. Data files were anonymized and linked with encrypted identification numbers to protect the privacy of the patients. Diagnostic codes are in the format of the International Classification of Disease, Ninth Revision, Clinical Modification (ICD-9-CM).

Ethics statement. The NHIRD encrypts patient's personal information to protect privacy (indexes included patients' name, personal ID, living address) and provides researchers with anonymous identification numbers associated with relevant claim information, including patients' sex, dates of birth, medical services utilized, and prescriptions. Patient consent is not required for accessing the NHIRD as described in detail previously (8). This study was approved by the Institutional Review Board of China Medical University Hospital (CMUH104-REC2-115). Our IRB specifically waived the consent requirement.

Sampled patients. For this retrospective case-control study, patients newly diagnosed with hyperlipidemia (ICD-9-CM code 272) between January 1, 2006 and December 31, 2011 were identified in the LHID as the case group and a comparison group of participants without hyperlipidemia. Hyperlipidemia involves abnormally elevated levels of one or more lipids and/or lipoproteins in the blood (e.g., triglyceride (TG) or cholesterol) and pathological lipid qualities such as elevated levels of low-density lipoprotein (LDL), which is the most common form of dyslipidemia. In this study, we included patients with a diagnosis of hyperlipidemia (ICD-9-CM codes 272.0, 272.1, 272.2,
272.3, and 272.4). The date of hyperlipidemia diagnosis was defined as the index date. We selected the non- hyperlipidemia patients from the LHID by 1:4 matching with the hyperlipidemia patient on a propensity score (12). The propensity score was calculated by a logistic regression to estimate the probability of the disease status given the baseline variables including gender, age, urbanization level, income, diabetes, hypertension, obesity, coronary artery disease, cirrhosis, and HIV infection. Figure 1 shows a flow chart of the selection procedure of the study participants.

In addition, the level of urbanization was divided into 4 levels based on the NHRI report (Level 1 was the highest level of urbanization and Level 7 was the lowest). Because only a few people lived in Levels 5-7, we grouped the least urbanized populations into Level 5). Cities, districts, and townships within which subjects were registered for insurance purposes were grouped into four levels of urbanization based on the population density (people $/ \mathrm{km}^{2}$ ). Level 1 indicates the most urbanized area, and level 4 indicates the least urbanized.

Antituberculosis medication exposure and comorbidities. TB diagnoses were peer reviewed and the patients that had used the medication, INH, RIF, ethambutol, pyrazinamide, and streptomycin, before the index date were classified as exposed to anti-TB medication. Medications were separated into six classes: INH, RIF, both INH and RIF, ethambutol, pyrazinamide, and streptomycin. According to total treatment duration (in days) and the quantity of anti-TB medication, the cumulative dose of each type of anti-TB medication was calculated for each user. Several well-known risk factors for hyperlipidemia were also selected, namely diabetes (ICD-9-CM 250), hypertension (ICD9-CM 401-405), obesity (ICD-9-CM 278), coronary artery disease (ICD-9-CM 410-414), cirrhosis (ICD-9-CM 571), and HIV infection (ICD-9-CM 795.71, V08, 042, 079.53), as comorbidities. Owing to restrictions in the database set of HIV drugs used in Taiwan, the use of only a few anti-HIV drugs (lamivudine, tenofovir disoproxil fumarate, didanosine, ritonavir, and saquinavir) was compared between the patients with hyperlipidemia and the controls. In addition, related studies that have applied the same diagnosis method and ICD-9 coding criteria have been published $(13,14)$

Statistical analysis. The proportional distributions of sex, age (20$49,50-64,65-74, \geq 75$ years), urbanization level (level 1 was the highest, and level 4 was the lowest), and income ( $<15,840,15,840$ 19,200 , and $\geq 19,200$ NTD) as well as the anti-TB and anti-HIV drug medication use and comorbidities of the treated group were compared with those of the control group. The standardized difference was used to quantify differences in mean or prevalence between the hyperlipidemia and non-hyperlipidemia groups for continuous or categorical variables, respectively (15). A value of standardized mean differences equal to 0.01 or less, indicates a negligible difference between means of hyperlipidemia and nonhyperlipidemia groups.

Univariable and multivariable unconditional logistic regression analyses were conducted to estimate the odds ratios (ORs) and 95\% confidence intervals (CIs) for the association between hyperlipidemia and anti-TB medication use. Adjusted ORs were also determined after we controlled for age, sex, anti-HIV drug use, and comorbidities, namely diabetes, hypertension, obesity, coronary artery disease, cirrhosis, and HIV infection. In additional analyses, the effect of the anti-TB medication cumulative duration and dosage were also estimated using logistic regression. All analyses were 


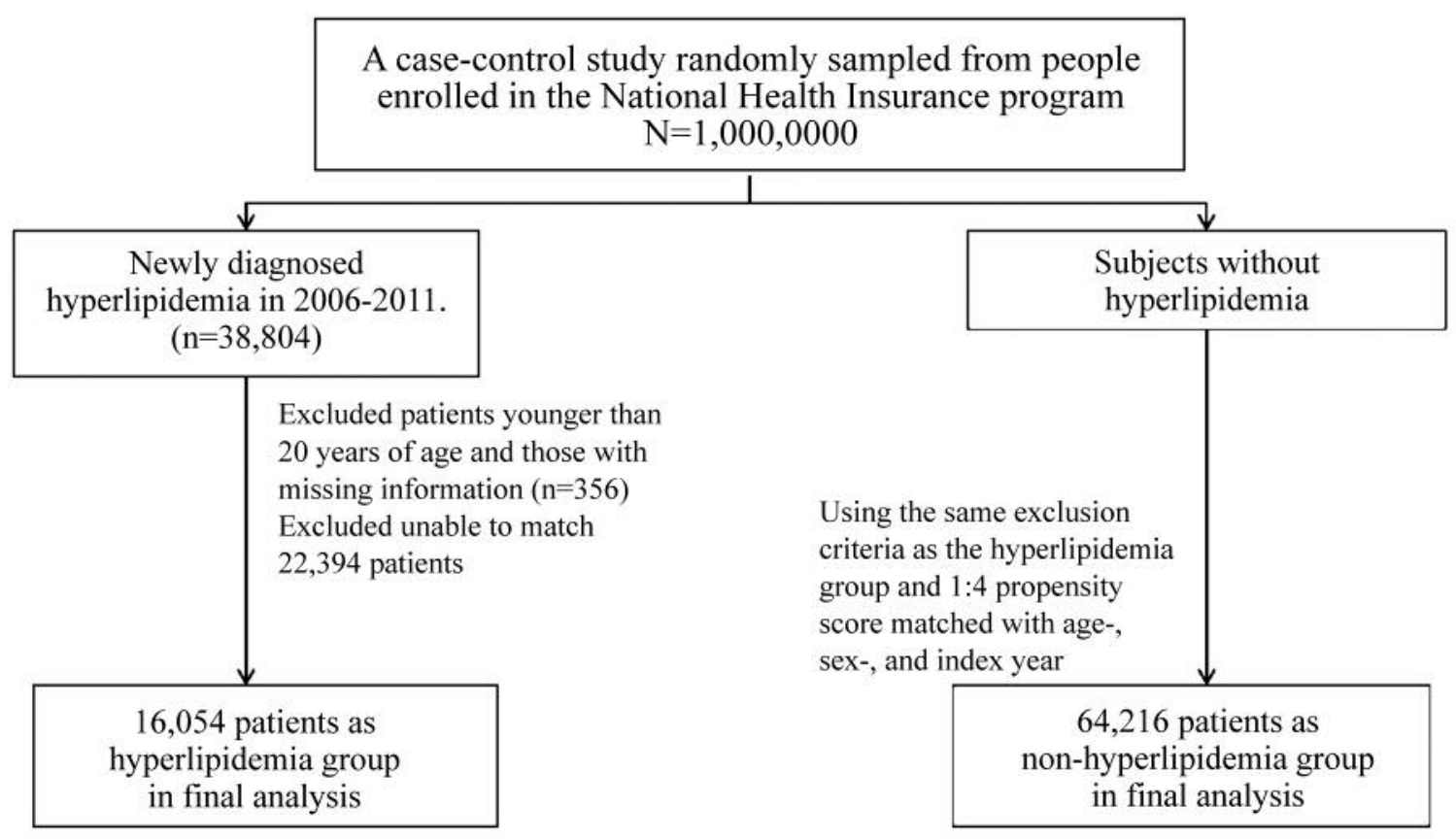

Figure 1. Process for selecting the participants in the two study groups.

conducted using SAS statistical software (Version 9.4 for Windows; SAS Institute, Inc., Cary, NC, USA), and all statistical tests were performed at a two-tailed significance level of 0.05 .

Ethical approval. Our study was exempt from full review by the Third Research Ethnics Committee of China Medical University and Hospital (IRB registration number: CMUH-104-REC2-115) on Apr. 23, 2015.

Informed consent. The NHIRD encrypts patient personal information to protect privacy and provides researchers with anonymous identification numbers associated with relevant claims information, including sex, date of birth, medical services received, and prescriptions. Therefore, patient consent is not required to access the NHIRD. This study was approved to fulfill the condition for exemption by the Institutional Review Board (IRB) of China Medical University (CMUH104-REC2-115). The IRB also specifically waived the consent requirement.

\section{Results}

Characteristics of the included patients. The case group comprised 16,054 patients with newly diagnosed hyperlipidemia, and the control group comprised 64,216 people without hyperlipidemia (Table I). Our study had more male than female patients $(49.7 \% v s .51 .1 \%)$, and were younger than 64 years of age $(77 \% v s .79 .3 \%)$. Compared with the control group, patients with hyperlipidemia tended to live in urban areas $(58.5 \% \mathrm{vs}$. $60.5 \%$ at urbanization levels 1 and 2) and had incomes of between 15,840-25,200 NTD
(47.6\% vs. $45.8 \%)$. The mean ages of the hyperlipidemia patients and controls were $53.5( \pm 14.3)$ and $53.0( \pm 13.9)$ years. INH use was significantly lower in the hyperlipidemia group than in the control group $(0.59 \%$ vs. $0.76 \%)$. Compared with the control group, the hyperlipidemia group was more likely to have diabetes, hypertension, coronary artery disease, cirrhosis, and HIV infection.

Association of hyperlipidemia with anti-tuberculosis users and covariates. Table II shows the crude and adjusted ORs of hyperlipidemia by anti-TB medication and comorbidities. Univariable unconditional logistic analysis revealed that compared with non-INH use, the OR of hyperlipidemia was 0.77 for $\mathrm{INH}$ use $(95 \% \mathrm{CI}=0.61-0.96)$. After adjustment for (1) age, sex, urbanization level, and income, (2) and anti-HIV drug medications, and (3) comorbidities of diabetes, hypertension, coronary artery disease, and cirrhosis, INH $(\mathrm{OR}=0.71$, 95\%CI0.57-0.88) was significantly associated with a decreased risk of hyperlipidemia. The adjusted OR of hyperlipidemia increased with income and was 1.23 for those with the lowest income compared with those with highest income (95\%CI=1.17-1.29). The patients living in the lower urbanized areas had a higher adjusted OR of hyperlipidemia compared with those living in the most urbanized areas. Anti-HIV drug use $(\mathrm{OR}=0.39,95 \% \mathrm{CI}=0.21-0.72)$ was significantly associated with a decreased risk of hyperlipidemia. Comorbidities of coronary artery disease and cirrhosis were associated with a significantly increased risk of hyperlipidemia. 
Table I. Baseline characteristics of the hyperlipidemia group and non-hyperlipidemia group.

\begin{tabular}{|c|c|c|c|c|c|}
\hline & \multicolumn{4}{|c|}{ Hyperlipidemia } & \multirow[b]{3}{*}{ Standard difference } \\
\hline & \multicolumn{2}{|c|}{$\begin{array}{c}\text { No } \\
\mathrm{N}=64,216\end{array}$} & \multicolumn{2}{|c|}{$\begin{array}{c}\text { Yes } \\
\mathrm{N}=16,054\end{array}$} & \\
\hline & $\mathrm{N}$ & $\%$ & $\mathrm{n}$ & $\%$ & \\
\hline \multicolumn{6}{|l|}{ Gender } \\
\hline Women & 32820 & 51.1 & 7984 & 49.7 & 0.03 \\
\hline Men & 31396 & 48.9 & 8070 & 50.3 & 0.03 \\
\hline \multicolumn{6}{|l|}{ Age group (year) } \\
\hline $20-49$ & 27521 & 42.9 & 6275 & 39.1 & 0.08 \\
\hline $50-64$ & 23348 & 36.4 & 6076 & 37.9 & 0.03 \\
\hline $65-74$ & 11052 & 17.2 & 3077 & 19.2 & 0.05 \\
\hline$\geq 75$ & 2295 & 3.57 & 626 & 3.90 & 0.02 \\
\hline Mean (SD) (year) * & $53.0(13.9)$ & & $53.5(14.3)$ & & 0.04 \\
\hline \multicolumn{6}{|l|}{ Urbanization level§ } \\
\hline 1 (highest) & 21231 & 33.1 & 4578 & 28.5 & 0.10 \\
\hline 2 & 17596 & 27.4 & 4808 & 30.0 & 0.06 \\
\hline 3 & 10999 & 17.1 & 2932 & 18.3 & 0.03 \\
\hline 4 (lowest) & 14390 & 22.4 & 3736 & 23.3 & 0.02 \\
\hline \multicolumn{6}{|l|}{ Income (NTD) } \\
\hline$<15,840$ & 12613 & 19.6 & 3502 & 21.8 & 0.05 \\
\hline $15,840-25,200$ & 29401 & 45.8 & 7642 & 47.6 & 0.04 \\
\hline$\geq 25,200$ & 22202 & 34.6 & 4910 & 30.6 & 0.09 \\
\hline \multicolumn{6}{|l|}{ Medications } \\
\hline Isoniazid & 490 & 0.76 & 94 & 0.59 & 0.02 \\
\hline Rifampin & 1756 & 2.73 & 410 & 2.55 & 0.01 \\
\hline Combine use of Isoniazid and Rifampin & 549 & 0.85 & 123 & 0.77 & 0.01 \\
\hline Ethambutol & 909 & 1.42 & 195 & 1.21 & 0.02 \\
\hline Pyrazinamide & 676 & 1.05 & 145 & 0.90 & 0.02 \\
\hline Streptomycin & 70 & 0.11 & 10 & 0.06 & 0.02 \\
\hline Anti-HIV drugs & 96 & 0.15 & 11 & 0.07 & 0.03 \\
\hline \multicolumn{6}{|l|}{ Baseline co-morbidities } \\
\hline Diabetes & 1665 & 2.59 & 478 & 2.98 & 0.02 \\
\hline Hypertension & 12568 & 19.6 & 3354 & 20.9 & 0.03 \\
\hline Obesity & 457 & 0.71 & 129 & 0.80 & 0.01 \\
\hline Coronary artery disease & 6247 & 9.73 & 2154 & 13.4 & 0.11 \\
\hline Cirrhosis & 10539 & 16.4 & 3195 & 19.9 & 0.09 \\
\hline HIV infection & 33 & 0.05 & 3 & 0.02 & 0.02 \\
\hline
\end{tabular}

Data are presented as the number of subjects in each group, with percentages given in parentheses. §Urbanization level was categorized by the population density of the residential area into four levels, with level 1 as the most urbanized and level 4 as the least urbanized.

Association of hyperlipidemia with cumulative duration and dosage of anti-tuberculosis medication use. We estimated the risk of hyperlipidemia according to the cumulative duration and dosage of anti-TB medication use (Table III). Among patients who used INH for 6 months or more, the adjusted ORs were $0.58(95 \% \mathrm{CI}=0.40-0.85)$. Compared to the non-RIF users, the risk of hyperlipidemia was $0.68(95 \% \mathrm{CI}=0.49-0.94)$ for those who used RIF for $>6$ months. INH and RIF dosages of $>32,700 \mathrm{mg}$ and $>50,400 \mathrm{mg}$, respectively, were also significantly associated with a decreased risk of hyperlipidemia $(\mathrm{INH}, \quad \mathrm{OR}=0.60, \quad 95 \% \mathrm{CI}=0.43-0.84 ; \quad \mathrm{RIF}, \quad \mathrm{OR}=0.63$, $95 \% \mathrm{CI}=0.44-0.91)$. Additionally, we determined that the dosage of the combination of INH and RIF use was associated with a lower risk of hyperlipidemia $(\mathrm{OR}=0.36,95 \% \mathrm{CI}=0.19$ 0.70 for $>33,600 \mathrm{mg}$ ). Similar results were observed for ethambutol and pyrazinamide use for the cumulative duration and dosage. Thus, a dose-dependent risk reduction of hyperlipidemia was observed in the patients using INH, RIF, and a combination of INH and RIF, with the ORs progressively decreasing as the cumulative dose was increased.

\section{Discussion}

We conducted a population-based case-control study by using data from the Taiwan NHI database to investigate the use of anti-TB medications, INH and RIF, and their 
Table II. Odds ratios and 95\% confidence intervals of hyperlipidemia associated with anti-tuberculosis and covariates.

\begin{tabular}{|c|c|c|c|c|}
\hline \multirow[b]{2}{*}{ Variable } & \multicolumn{2}{|c|}{ Crude } & \multicolumn{2}{|c|}{ Adjusted ${ }^{\dagger}$} \\
\hline & OR & $(95 \% \mathrm{CI})$ & OR & $(95 \% \mathrm{CI})$ \\
\hline \multicolumn{5}{|l|}{ Urbanization level ${ }^{\S}$} \\
\hline 1 (highest) & 1 & (Reference) & 1 & (Reference) \\
\hline 2 & 1.27 & $(1.21,1.33)^{* * *}$ & 1.21 & $(1.16,1.27)^{* *}$ \\
\hline 3 & 1.24 & $(1.17,1.30)^{* * *}$ & 1.18 & $(1.12,1.25)^{* * *}$ \\
\hline 4 (lowest) & 1.20 & $(1.15,1.26)^{* * *}$ & 1.13 & $(1.08,1.19)^{* * *}$ \\
\hline \multicolumn{5}{|l|}{ Income (NTD) } \\
\hline$<15,840$ & 1.26 & $(1.20,1.32)^{* * *}$ & 1.23 & $(1.17,1.29)^{* * *}$ \\
\hline $15,840-25,200$ & 1.18 & $(1.13,1.22)^{* * *}$ & 1.13 & $(1.08,1.18)^{* * *}$ \\
\hline$\geq 25,200$ & 1 & (Reference) & 1 & (Reference) \\
\hline \multicolumn{5}{|l|}{ Medications } \\
\hline Isoniazid & 0.77 & $(0.61,0.96)^{*}$ & 0.71 & $(0.57,0.88)^{* * *}$ \\
\hline Rifampin & 0.93 & $(0.84,1.04)$ & - & - \\
\hline Combine use of Isoniazid and Rifampin & 0.90 & $(0.74,1.09)$ & - & - \\
\hline Ethambutol & 0.86 & $(0.73,1.00)$ & - & - \\
\hline Pyrazinamide & 0.86 & $(0.72,1.03)$ & - & - \\
\hline Streptomycin & 0.57 & $(0.30,1.11)$ & - & - \\
\hline Anti-HIV drug & 0.46 & $(0.25,0.86)^{*}$ & 0.39 & $(0.21,0.72)^{* *}$ \\
\hline \multicolumn{5}{|l|}{ Baseline co-morbidities } \\
\hline Diabetes & 1.15 & $(1.04,1.28)^{* *}$ & 1.02 & $(0.91,1.13)$ \\
\hline Hypertension & 1.09 & $(1.04,1.13)^{* * *}$ & 0.96 & $(0.91,1.02)$ \\
\hline Obesity & 1.13 & $(0.93,1.38)$ & - & - \\
\hline Coronary artery disease & 1.44 & $(1.37,1.52)^{* * *}$ & 1.43 & $(1.35,1.51)^{* * *}$ \\
\hline Cirrhosis & 1.27 & $(1.21,1.32)^{* * *}$ & 1.24 & $(1.19,1.30)^{* * *}$ \\
\hline HIV infection & 0.36 & $(0.11,1.19)$ & - & - \\
\hline
\end{tabular}

$\dagger$ Adjusted for age, sex, urbanization level, income (NTD), and medications of anti-HIV drugs as well as comorbidities of diabetes, hypertension, coronary artery disease, and cirrhosis; ${ }^{*} p<0.05 ; * * p<0.01 ; * * p<0.001$.

correlation with hyperlipidemia, with adjustments for hyperlipidemia-related comorbidities. More than $99 \%$ of Taiwan residents' complete medicinal records have been included in the NHI database since 1996 (16). We collected a large sample and detected statistical differences between anti-TB medication users and nonusers by using this comprehensive health surveillance research system. A significant $29 \%$ decrease in hyperlipidemia incidence was observed in the INH users compared with the nonusers. In addition, these significant decreases were more apparent in those who had used INH and RIF for a cumulative duration of $>6$ months (adjusted ORs of 0.58 and 0.68 , compared to the non-users, respectively). A dose-dependent decreased risk of hyperlipidemia was observed in the INH and RIF users, with the ORs progressively decreasing as the cumulative dose was increased. These results suggest that INH and RIF users have a reduced risk of hyperlipidemia.

In Taiwan, approximately $45 \%$ of prescriptions are not treated in accordance with standard protocols, and secondline drugs are prescribed (5). Because of medical treatment variations among individuals, clinical physicians may integrate their clinical experience, expertise, professional knowledge, and patient conditions to provide suitable treatment. INH, RIF, or a combination of INH and RIF (Rifinah) are the most commonly used anti-TB chemotherapeutic treatments (5). INH and RIF are the most effective drugs for preventing drug resistance, followed by ethambutol and pyrazinamide. Long-term use or higher doses of INH and RIF have been considered for treating multidrugresistant (MDR)-TB and extensively drug-resistant (XDR)TB. According to the WHO, 3.7\% of new TB patients and $20 \%$ of previously treated TB patients are estimated to have MDR-TB (1). However, several reports have found that these drugs may be associated with the side effects of liver function abnormality or even hepatocellular carcinoma (HCC) in patients with liver cirrhosis $(7,8)$. In most cases, acute liver failure is associated with INH and RIF treatment within 10 days of the beginning of therapy (7). The hepatotoxicity of INH is caused by its metabolization to acetyl hydrazine and hydrazine by cytochrome P450 (CYP450) (17). The toxicity is enhanced by RIF, a strong CYP450 inducer, particularly of CYP3A4, when RIF is coadministered. Hepatic cell death may be caused by the depletion of glutathione by the high reactivity of hydrazine and can lead to chronic liver disease or even HCC. However, few cases of liver failure induced by treatment with INH and RIF have been reported. 
in vivo $32: 47-54(2018)$

Table III. Odds ratio and 95\% confidence intervals (CI of hyperlipidemia associated with cumulative duration and dosage of antituberculosis medication use.

\begin{tabular}{|c|c|c|c|c|c|}
\hline Variables & $\begin{array}{l}\text { Case number/ } \\
\text { control number }\end{array}$ & $\begin{array}{l}\text { Crude } \\
\text { odds ratio }\end{array}$ & $(95 \% \mathrm{CI})$ & $\begin{array}{l}\text { Adjusted } \\
\text { odds ratio }\end{array}$ & $(95 \% \mathrm{CI})$ \\
\hline \multicolumn{6}{|l|}{ Isoniazid } \\
\hline Non-use of Isoniazid & $16054 / 64216$ & 1.00 & (reference) & 1.00 & (reference) \\
\hline$\leq 6$ months & $62 / 288$ & 0.86 & $(0.65,1.13)$ & 0.79 & $(0.60,1.04)$ \\
\hline$>6$ months & $32 / 202$ & 0.63 & $(0.44,0.92)^{*}$ & 0.58 & $(0.40,0.85)^{* *}$ \\
\hline$\leq 32,700 \mathrm{mg} \S$ & $53 / 240$ & 0.88 & $(0.66,1.19)$ & 0.82 & $(0.61,1.10)$ \\
\hline$>32,700 \mathrm{mg} \S$ & $41 / 250$ & 0.66 & $(0.47,0.91)^{*}$ & 0.60 & $(0.43,0.84)^{* *}$ \\
\hline \multicolumn{6}{|l|}{ Rifampin } \\
\hline Non-use of Rifampin & $16054 / 64216$ & 1.00 & (reference) & 1.00 & (reference) \\
\hline$\leq 6$ months & $366 / 1517$ & 0.96 & $(0.86,1.08)$ & 0.93 & $(0.83,1.04)$ \\
\hline$>6$ months & $44 / 239$ & 0.74 & $(0.53,1.02)$ & 0.68 & $(0.49,0.94)^{*}$ \\
\hline$\leq 50,400 \mathrm{mg} \S$ & $376 / 1558$ & 0.96 & $(0.86,1.08)$ & 0.93 & $(0.83,1.04)$ \\
\hline$>50,400 \mathrm{mg} \S$ & $34 / 198$ & 0.69 & $(0.48,0.99)^{*}$ & 0.63 & $(0.44,0.91)^{*}$ \\
\hline \multicolumn{6}{|c|}{ Combine use of Isoniazid and Rifampin } \\
\hline $\begin{array}{l}\text { Non-use of combine use of } \\
\text { Isoniazid and Rifampin }\end{array}$ & $38124 / 152555$ & 1.00 & (reference) & 1.00 & (reference) \\
\hline$\leq 6$ months & $74 / 318$ & 0.93 & $(0.72,1.20)$ & 0.84 & $(0.65,1.09)$ \\
\hline$>6$ months & $49 / 231$ & 0.85 & $(0.62,1.15)$ & 0.78 & $(0.57,1.07)$ \\
\hline$\leq 33,600 \mathrm{mg} \S$ & $113 / 450$ & 1.00 & $(0.82,1.23)$ & 0.92 & $(0.75,1.13)$ \\
\hline$>33,600 \mathrm{mg} \S$ & $10 / 99$ & 0.40 & $(0.21,0.77)^{* *}$ & 0.36 & $(0.19,0.70)^{* *}$ \\
\hline Ethambutol & $16054 / 64216$ & 1.00 & (reference) & 1.00 & (reference) \\
\hline$\leq 6$ months & $107 / 476$ & 0.90 & $(0.73,1.11)$ & 0.84 & $(0.68,1.04)$ \\
\hline$>6$ months & $88 / 433$ & 0.81 & $(0.65,1.02)$ & 0.75 & $(0.59,0.94)^{*}$ \\
\hline$\leq 134,600 \mathrm{mg} \S$ & $102 / 450$ & 0.91 & $(0.73,1.12)$ & 0.86 & $(0.69,1.06)$ \\
\hline$>134,600 \mathrm{mg} \S$ & $93 / 459$ & 0.81 & $(0.65,1.01)$ & 0.74 & $(0.59,0.93)^{* *}$ \\
\hline Pyrazinamide & $16054 / 64216$ & 1.00 & (reference) & 1.00 & (reference) \\
\hline$\leq 6$ months & $133 / 559$ & 0.95 & $(0.79,1.15)$ & 0.88 & $(0.73,1.07)$ \\
\hline$>6$ months & $12 / 117$ & 0.41 & $(0.23,0.74)^{* *}$ & 0.37 & $(0.21,0.68)^{* *}$ \\
\hline$\leq 151,200 \mathrm{mg} \S$ & $83 / 358$ & 0.93 & $(0.73,1.18)$ & 0.87 & $(0.68,1.11)$ \\
\hline$>151,200 \mathrm{mg}^{\S}$ & $62 / 318$ & 0.78 & $(0.59,1.02)$ & 0.71 & $(0.54,0.93)^{*}$ \\
\hline Streptomycin & $16054 / 64216$ & 1.00 & (reference) & 1.00 & (reference) \\
\hline$\leq 6$ months & $10 / 60$ & 0.67 & $(0.34,1.30)$ & 0.61 & $(0.31,1.19)$ \\
\hline$>6$ months & $0 / 10$ & - & - & - & - \\
\hline$\leq 185,600 \mathrm{mg} \S$ & $4 / 43$ & 0.37 & $(0.13,1.04)$ & 0.34 & $(0.12,0.94)^{*}$ \\
\hline$>185,600 \mathrm{mg} \S$ & $6 / 27$ & 0.89 & $(0.37,2.15)$ & 0.84 & $(0.35,2.04)$ \\
\hline
\end{tabular}

$\S$ Cumulative dosage by median. ${ }^{\dagger}$ Adjusted for age, sex, urbanization level, income (NTD), medications of ethambutol, pyrazinamide, streptomycin, and anti-HIV drugs as well as comorbidities of diabetes, hypertension, obesity, coronary artery disease, cirrhosis, and HIV infection; * $p<0.05$; $* * p<0.01 ; * * * p<0.001$

The liver is critical in lipid metabolism. It absorbs serumfree fatty acids and manufactures, stores, and transports lipid metabolites (18). Lipids, mainly TGs, occur in hepatocytes and represent a hallmark feature of the pathogenesis of nonalcoholic fatty liver disease (18). The clinical implication of hyperlipidemia or the combination of lipid abnormalities, including high serum LDL-cholesterol, TG, and low serum high density lipoprotein-cholesterol levels. Hyperlipidemia is crucial in the progression of cardiovascular diseases
(CVDs), including coronary artery disease, atherosclerosis, deep vein thrombosis, and cerebrovascular disease (19). Currently, the association among lipids, anti-TB medications, and CVD risk remains unclear.

Several reports have indicated that chronic liver disease and liver cancer may affect blood fat levels $(20,21)$. One study found that, in 40 liver cancer cases, serum TG levels were decreased by $28.8 \%$. This phenomenon may be explained by the relationship between cytokines and lipids 
(22). Tumor cells produce cytokines, such as tumor necrosis factor $\alpha$, interleukins 1 and 6, and interferon $\alpha$ (23), which control lipid storage (24). In addition, hepatic associated diseases lead to blockage of cholesterol esterification and evacuation, thus changing plasma cholesterol levels (25).

Animals' studies have shown that rats exposed to INH and RIF combination treatment at $50 \mathrm{mg} / \mathrm{kg} \mathrm{wt} /$ day of each drug, which is a higher dosage than the therapeutic dose for human use, had significantly increased liver and serum cholesterol levels compared with an untreated group. In addition, liver TGs, but not serum TGs, increased significantly in the treated group (26). Moreover, serum phospholipids were increased only in the treated group, and the liver phospholipid content significantly decreased. It may originate from decreases in liver lipoprotein synthesis, resulting in lipid mobilization impairment and thus the accumulation of TG in the liver. Some reports have shown that higher liver and serum cholesterol levels were observed in patients exposed to longterm INH and RIF combination treatment; these increased levels may have been attributable to the inhibition of bile secretion, leading to cholesterol accumulation in the liver (27). Additionally, RIF has been reported to cause hyperlipidemia in TB patients (28). These studies have thus revealed that animals treated with a combination of INH and RIF have lipid profile alterations, and liver lipid and serum lipid levels are not fully interchangeable according to the observations from these studies.

Activation of human pregnane $\mathrm{X}$ receptor (PXR; NR1I2) induces human CYP3A4 in drug metabolism and inhibits cholesterol $7 \alpha$-hydroxylase (CYP7A1) in bile acid synthesis (29). RIF, a strong PXR inducer, inhibits bile acid synthesis and has been used for cholestatic disease treatment (30). Activation of PXR by RIF accelerates PXR interaction with the hepatocyte nuclear factor $4 \alpha(\mathrm{HNF} 4 \alpha, N R 2 A 1)$ and blocks strong interaction with a positive controller, peroxisome proliferator-activated receptor coactivator 1alpha with HNF4 $\alpha$ (29). Consequently, RIF inhibits bile acid synthesis by inhibiting $C Y P 7 A 1$ gene transcription as well as bile acid synthesis and thus prevents cholestasis.

INH is the preferred drug for treating latent TB infection (LTBI). Historically, LTBI treatment has been considered a prophylaxis; in other words, INH alone is administered for 9 months to reduce the risk of active TB. A previous study showed that INH treatment was significantly associated with a decrease of total cholesterol and ApoB levels (31). This indicates that LTBI treatment may affect lipid metabolism. Regardless of INH hepatotoxicity, INH might be prescribed less frequently to people with known hyperlipidemia. Thus, a stronger association was observed with INH than with RIF.

This is the first study examining an Asian population to use a population-based nationwide database to evaluate the effect of INH and RIF use on hyperlipidemia development. However, our study had several limitations. First, information regarding lifestyle, such as behavior, smoking status, alcohol consumption, environmental exposure, body weight or body mass index, and family history of hyperlipidemia, is unavailable in the LHIRD. Some of these factors may be contributory and confounding because they may increase the risk of hyperlipidemia. Second, we were unable to contact the patients directly to obtain additional information because the data were anonymous. The claims data in the LHIRD are used primarily for administrative billing purposes. Third, according to the WHO, the incidence of MDR-TB and XDR-TB is increasing globally (1) because of limitations in the clinical laboratory diagnostic methodology and the absence of exact laboratory data to confirm TB pathogen types. Physicians might thus increase the treatment dose or duration of first-line anti-TB medications to treat the disease (5). Resistance conditions differ among patients, and physicians might have individual preferences regarding the prescription of medications. In this study, we evaluated $>6$ months of exposure to anti-TB medications to minimize bias associated with these factors. Fourth, the LHIRD lacks complete information on the ChildPugh classification of liver disease, which might correlate highly with the risk of hyperlipidemia. Fifth, from our database, we could not differentiate the active or latent phase of TB infection. Monotherapy can be used only for latent infection. Once active disease is present, multi-drugs regimen, and generally three or four drugs, must be used simultaneously (5). In addition, asymptomatic hyperlipidemia is usually underdiagnosed. Finally, no data on dietary effects were available in this study; dietary intake types may contribute to the control of lipids in the body.

As the treatment of hyperlipidemia should follow the guidelines given by the National Cholesterol Education Program, some efforts should be made on several risk factors that are strongly related to CVD (e.g., smoking, hypertension, diabetes mellitus, lifestyle, dietary intervention, physical activity, or medications). INH and RIF do not seem to be clinical medications that are strongly correlated with hyperlipidemia.

In conclusion, our study results may provide epidemiologic evidence to support the possible association between longterm and high-dose use of INH, RIF, and combined INH and RIF and a decreased risk of hyperlipidemia. Other risk factors and ethnic factors should be considered to evaluate the decreased risk of hyperlipidemia under these anti-TB treatments and their effects on CVD. In addition, large population-based studies are required to confirm our observations and clarify the possible mechanisms by which these anti-TB medications reduce blood lipid levels.

\section{Conflicts of Interest}

The Authors have declared that no competing interests exist. 


\section{Acknowledgements}

This study was supported mainly by the Ministry of Science and Technology, Taiwan, R.O.C. (MOST105-2320-B-039-031) and China Medical University Hospital, Taichung, Taiwan (DMR-106066) to Prof Lim, and partially supported by the Taiwan Ministry of Health and Welfare Clinical Trial and Research Center of Excellence (MOHW106-TDU-B-212-113004), China Medical University Hospital, Academia Sinica Taiwan Biobank Stroke Biosignature Project (BM10601010036), Taiwan Clinical Trial Consortium for Stroke (MOST106-2321-B-039-005).

\section{References}

1 World Health Organization: Global tuberculosis report 2016 World Health Organization, 2016.

2 Lin YT, Wu PH, Lin CY, Lin MY, Chuang HY, Huang JF, Yu ML and Chuang WL: Cirrhosis as a risk factor for tuberculosis infection-a nationwide longitudinal study in Taiwan. Am J Epidemiol 180: 103-110, 2014.

3 Centers for Disease Control. Taiwan Tuberculosis Control Report 2013. ISSN 1996-8221, Annual April, 2014. Centers for Disease Control, Ministry of Health and Welfare, R.O.C. (Taiwan).

4 Churchyard GJ, Fielding KL, Lewis JJ, Coetzee L, Corbett EL, Godfrey-Faussett P, Hayes RJ, Chaisson RE and Grant AD: A trial of mass isoniazid preventive therapy for tuberculosis control. N Engl J Med 370: 301-310, 2014.

5 Wang SI, Shen GH, Shi HC and Chiou SJ: Prescription patterns for tuberculosis treatment and adherence to treatment guidelines: a population-based study in Taiwan. Pharmacoepidemiol Drug Saf 23: 1273-1280, 2014.

6 Shih TY, Young TH, Lee HS, Hsieh CB and Hu OY: Protective effects of kaempferol on isoniazid- and rifampicin-induced hepatotoxicity. AAPS J 15: 753-762, 2013.

7 Forget EJ and Menzies D: Adverse reactions to first-line antituberculosis drugs. Expert Opin Drug Saf 5: 231-249, 2006.

8 Lim YP, Lin CL, Hung DZ, Lin YN and Kao CH: Antituberculosis treatments and risk of hepatocellular carcinoma in tuberculosis patients with liver cirrhosis: a population-based casecontrol study. Eur J Clin Microbiol Infect Dis 34: 479-485, 2015.

9 Tasduq SA, Kaiser P and Sharma SC: Potentiation of isoniazidinduced liver toxicity by rifampicin in a combinational therapy of antitubercular drugs (rifampicin, isoniazid and pyrazinamide) in Wistar rats: A toxicity profile study. Hepatol Res 37: 845-853, 2007.

10 Ramappa V and Aithal GP: Hepatotoxicity related to antituberculosis drugs: mechanisms and management. J Clin Exp Hepatol 3: 37-49, 2013.

11 Chen X, Zhang C, Wang H, Xu J, Duan ZH, Zhang Y, Yu T, Wei $\mathrm{W}, \mathrm{Xu} \mathrm{DX}$ and $\mathrm{Xu}$ JM: Altered integrity and decreased expression of hepatocyte tight junctions in rifampicin-induced cholestasis in mice. Toxicol Appl Pharmacol 240: 26-36, 2009.

12 Parsons LS, Ovation Research Group, Seattle, Washington: Performing a 1: $\mathrm{N}$ case-control match on propensity score. SUGI 29: 1-11, 2001.

13 Shen $\mathrm{CH}$, Chou CH, Liu FC, Lin TY, Huang WY and Wang YC: Association between tuberculosis and Parkinson disease: A nationwide, population-based cohort study. Medicine 95: e2883, 2016.
14 Lim YP, Lin CL, Lin YN, Ma WC, Hung DZ and Kao CH: Tamoxifen treatment and the reduced risk of hyperlipidemia in Asian patients with breast cancer: A population-based cohort study. Clin Breast Cancer 15: 294-300, 2015.

15 Austin PC: Balance diagnostics for comparing the distribution of baseline covariates between treatment groups in propensityscore matched samples. Stat Med 28: 3083-3107, 2009.

16 Cheng TM: Taiwan's new national health insurance program: genesis and experience so far. Health Aff 22: 61-76, 2003.

17 Preziosi P: Isoniazid: metabolic aspects and toxicological correlates. Curr Drug Metab 8: 839-851, 2007.

18 Jiang JT, Xu N, Zhang XY and Wu CP: Lipids changes in liver cancer. J Zhejiang Univ Sci B 8: 398-409, 2007.

19 Rosenson RS, Davidson MH, Hirsh BJ and Gaudet D: Genetics and causality of triglyceride-rich lipoproteins in atherosclerotic cardiovascular disease. J Am Coll Cardiol 64: 2525-2540, 2014.

20 Cicognani C, Malavolti M, Morselli-Labate AM, Sama C and Barbara L: Serum lipid and lipoprotein patterns in patients with liver cirrhosis and chronic active hepatitis. Arch Intern Med 157: 792-796, 1997.

21 Motta M, Giugno I, Ruello P, Pistone G, Di-Fazio I and Malaguarnera M: Lipoprotein (a) behaviour in patients with hepatocellular carcinoma. Minerva Med 92: 301-305, 2001.

22 Malaguarnera M, Trovato G, Restuccia S, Giugno I, Franze CM, Receputo G, Siciliano R, Motta M and Trovato BA: Treatment of nonresectable hepatocellular carcinoma: review of the literature and meta-analysis. Adv Ther 11: 303-319, 1994.

23 Marra F and Tacke F: Roles for chemokines in liver disease. Gastroenterology 147: 577-594, 2014.

24 Wang Y, Wang H, Hegde V, Dubuisson O, Gao Z, Dhurandhar $\mathrm{NV}$ and Ye J: Interplay of pro- and anti-inflammatory cytokines to determine lipid accretion in adipocytes. Int J Obes 37: 14901498, 2013.

25 Cohen DE and Fisher EA: Lipoprotein metabolism, dyslipidemia, and nonalcoholic fatty liver disease. Semin Liver Dis 33: 380-388, 2013.

26 Pal R, Rana SV, Vaiphei K and Singh K: Isoniazid-rifampicin induced lipid changes in rats. Clin Chim Acta 389: 55-60, 2008.

27 Rana SV, Pal R, Vaiphie K and Singh K: Effect of different oral doses of isoniazid-rifampicin in rats. Mol Cell Biochem 289: 3947, 2006.

28 Khogali AM, Chazan BI, Metcalf VJ and Ramsay JH: Hyperlipidaemia as a complication of rifampicin treatment. Tubercle 55: 231-233, 1974.

$29 \mathrm{Li} \mathrm{T}$ and Chiang JY: Mechanism of rifampicin and pregnane $\mathrm{X}$ receptor inhibition of human cholesterol 7 alpha-hydroxylase gene transcription. Am J Physiol Gastrointest Liver Physiol 288: G74-84, 2005.

30 Zollner G and Trauner M: Nuclear receptors as therapeutic targets in cholestatic liver diseases. Br J Pharmacol 156: 7-27, 2009.

31 Albanna AS, Bachmann K, White D, Valiquette C and Menzies D: Serum lipids as biomarkers for therapeutic monitoring of latent tuberculosis infection. Eur Respir J 42: 547-550, 2013.

Received November 2, 2017

Revised November 21, 2017 Accepted November 23, 2017 